\title{
lethal of scute, a proneural gene, participates in the specification of muscle progenitors during Drosophila embryogenesis
}

\author{
Ana Carmena, ${ }^{1}$ Michael Bate, ${ }^{2}$ and Fernando Jiménez ${ }^{1,3}$ \\ ${ }^{1}$ Centro de Biología Molecular "Severo Ochoa" (C.S.I.C.-U.A.M.), Universidad Autónoma, 28049 Madrid, Spain; \\ ${ }^{2}$ Department of Zoology, University of Cambridge, Cambridge CB2 3EJ, UK
}

The mechanisms that underlie the segregation of muscle founder cells in the Drosophila embryo are undefined. We show that the proneural gene lethal of scute (l'sc) is expressed in clusters of cells in the somatic mesoderm, from which individual muscle progenitors are singled out by progressive restriction of l'sc expression. Coexpression of $l$ 'sc and $S 59$ (a putative muscle identity gene) in a subset of muscle progenitors shows that muscle founders are produced by division of muscle progenitors. In neurogenic mutant embryos the restriction of l'sc expression fails and all cells in a cluster coexpress l'sc and S59. Loss-of-function and overexpression phenotypes indicate a role for $l$ 'sc in the segregation of muscle progenitors and the formation of the muscle pattern.

[Key Words: lethal of scute; Drosophila; muscle progenitors; myogenesis; neurogenesis]

Received May 25, 1995; revised version accepted August 8, 1995.

During development in Drosophila, cells of the embryonic mesoderm that have entered the myogenic pathway are recruited to contribute to unique elements in the muscle pattern. Each muscle fibre is a highly individual structure, with a characteristic size, shape, insertion sites, and innervation (for review, see Bate 1993). The formation of this pattern requires that subsets of myoblasts gain access to the information required to make particular muscles. We envisage this happening as a result of a process of information transfer, which occurs in two essential steps. First, the pattern is seeded by the specification and segregation in the mesoderm of a number of unique cells, each of which is the founder of a distinct muscle (Bate 1990; Dohrmann et al. 1990). Second, in a nonspecific phase of fusion, founder cells recruit neighboring myoblasts to form the syncytial precursors of mature muscles.

Several observations support this model of muscle patterning. First, a growing number of genes have been identified that are expressed in subsets of mesodermal cells and appear to mark muscle founder cells (Dohrmann et al. 1990; Bourgouin et al. 1992; Bate 1993). These genes encode putative transcription factors, and they are expressed first in small numbers of mesodermal cells and later in subsets of muscles. As the initially expressing cells fuse with their neighbors, these cells are recruited

\footnotetext{
${ }^{3}$ Corresponding author.
}

to expression; in the absence of fusion, this recruitment does not occur (Rushton et al. 1995). Moreover, in mutant embryos where cell fusion fails to occur, the prospective muscle-forming cells appear to be of two kinds: a relatively undifferentiated set of myoblasts and a smaller set of single cells that express markers like S59 and vestigial $(\mathrm{vg})$ (Dohrmann et al. 1990; Williams et al. 1991; Bate et al. 1993|, which are normally characteristic of subsets of the embryonic muscles. In the mutant embryos, these latter cells often succeed in attaching to the epidermis and extending processes that span epidermal domains typical of the muscles that would express these genes in normal embryos (Rushton et al. 1995). Thus, in embryos where fusion fails, a special class of myoblasts is revealed, which appears to have the information required to seed the formation of particular muscles. We conclude that it is myoblasts of this class that act as the founders of individual muscles in wild-type embryos.

$S 59$ is the type example of genes that mark myoblasts of this class. It is first expressed in a consistent pattern of cells in the mesoderm of stage 11/12 embryos and then in a subset of the forming muscles in each segment, each of these being formed by the fusion of nonexpressing myoblasts with the original S59-expressing cells (Dohrmann et al. 1990). Although there are, as yet, no reported mutations in 559, the phenotype of nonfusion mutants strongly suggests that $S 59$ marks a special class of myoblasts required for the formation of 559 -expressing muscles. In such mutants 559 -expressing muscles are repre- 
sented by single 559 -expressing cells, which synthesize contractile proteins, are in appropriate positions, and span the territories normally occupied by $S 59$-expressing muscles (Rushton et al. 1995). S59 thus marks a subset of myoblasts that have the information required for the appropriate positioning and differentiation of 559 -expressing muscles.

The analogy in Drosophila between the formation of muscle founder cells in the embryonic mesoderm and the formation of neuroblasts in the neuroectoderm has been noted before (Bate 1990; Corbin et al. 1991). In both cases, single cells are in some way selected from their neighbors and assigned unique properties that seed the formation of a pattern. Furthermore, genetic evidence shows that founder cells and neuroblasts are both selected from larger groups of equivalent cells by mechanisms that require the functions encoded by the neurogenic genes. In embryos that are mutant for neurogenic genes, both the singling out of neuroblasts in the ectoderm and of founder cells in the mesoderm is deranged. In such mutant embryos, groups of neighboring ectodermal cells all form neuroblasts (for review, see CamposOrtega 1993) and enlarged clusters of cells in the mesoderm express founder cell marker genes (Corbin et al. 1991; Bate et al. 1993).

In the neuroectoderm, the groups of equivalently competent cells are revealed by the expression of proneural genes, which include members of the achaete-scute complex (AS-C) (for review, see Campos-Ortega 1993). Members of the AS-C, which encode transcription factors of the bHLH class, are expressed in clusters of ectodermal cells from which single neural progenitor cells will segregate. The absence of proneural gene functions leads to a loss of neural progenitors. So far the mechanism that demarcates groups of cells in the somatic mesoderm from which muscle founders will form is unknown. In vertebrates, a central role in myogenesis is ascribed to the bHLH genes of the MyoD family (Davis et al. 1987), and there are similarities in the function of these genes in the initiation of muscle development and the role of the proneural genes in Drosophila neurogenesis (Jan and Jan 1993). The elements of the myogenic pathway in Drosophila are as yet incompletely defined although it seems clear that nautilus (nau) (Michelson et al. 1990; Paterson et al. 1991), the single MyoD family member so far identified in the fly, does not have the central role in myogenesis that is attributed to it in vertebrates. nau is one of a diverse set of genes encoding putative transcription factors (including $S 59$ and $v g$ ) each of which is expressed in small numbers of mesodermal cells prior to myogenesis and in a subset of the differentiating muscles thereafter. These different factors may have a role in assigning distinctive properties to individual muscles in the set, but it is uncertain whether they are responsible for initiating more general features of muscle differentiation. Although, in this context, $D-M E F 2$ is known to be required generally in cells of the myogenic lineage for the expression of muscle myosin and the fusion of myoblasts (Bour et al. 1995; Lilly et al. 1995), the common features of the myogenic pathway, which, for different muscles, regulate the events leading to the segregation of founder cells are not known. In this paper, we report an investigation of the mesodermal expression and function in the Drosophila embryo of lethal of scute (I'sc), one of the proneural genes of the AS-C. Our results show that l'sc is expressed in well-defined clusters of cells in the somatic mesoderm, from which muscle founder cells segregate. Identified founder cells are consistently derived from specific groups of l'sc-expressing cells. In addition, loss-of-function in l'sc leads to a loss of specific muscles and muscle founder cells, whereas overexpression of l'sc causes muscles and founder cells to be duplicated. Taken together, our results indicate that l'sc plays an important role in the initiation of muscle patterning in Drosophila.

\section{Results}

Wild-type expression of l'sc

We have looked for the expression of proneural genes in the embryonic mesoderm. Although we do not see any mesodermal expression of achaete or scute, l'sc is clearly expressed in a characteristic pattern from stage 10 onward. The expression of l'sc in the mesoderm is transient and occurs in a very dynamic pattern (Fig. 1). From late stage 10 until stage 12, we find expression in at least 19 clusters in each hemisegment, whose size and location we have charted and mapped in Figure 2. The first clusters of expressing cells appear prior to the formation of the tracheal pits (Fig. 2, nos. 1-4), with others appearing in a rapid sequence during stage 11 as numbered. There is some segment specificity in the pattern, with cluster 18 being larger and expressing higher levels of l'sc in thoracic than in abdominal segments (Fig. ID). Cluster 19 is exclusive to thoracic segments. The evolution of these two clusters and of additional l'sc-expressing cells, which appear at late stage 11 and are also restricted to the thoracic segments, has not been analyzed further. In all other clusters examined, one cell accumulates higher levels of protein than the other members of the group. Usually, this cell comes to occupy a position closest to the ectoderm (nervous system or epidermis, depending on position). In most cases (clusters 2-9; 11-12; 14-16), l'sc expression seems to be gradually restricted to that single cell. Later, expression is lost from this cell as well. In the case of clusters $1,10,13$, and 17 , the restriction to a single cell, before ultimate loss of expression, is not so reliably observed. In the case of groups 3 and 12, there is some cell movement during the phase of 1 'sc expression. Thus, the single l'sc-expressing cell derived from group 3 migrates posteriorly and dorsally to lie adjacent to the cells of group 12. Group 12 itself moves dorsally before expression becomes restricted to a single cell.

l'sc-expressing cells are drawn from the twist-expressing mesodermal domain

To determine the location of l'sc-expressing cells within the mesodermal primordium, we double stained em- 


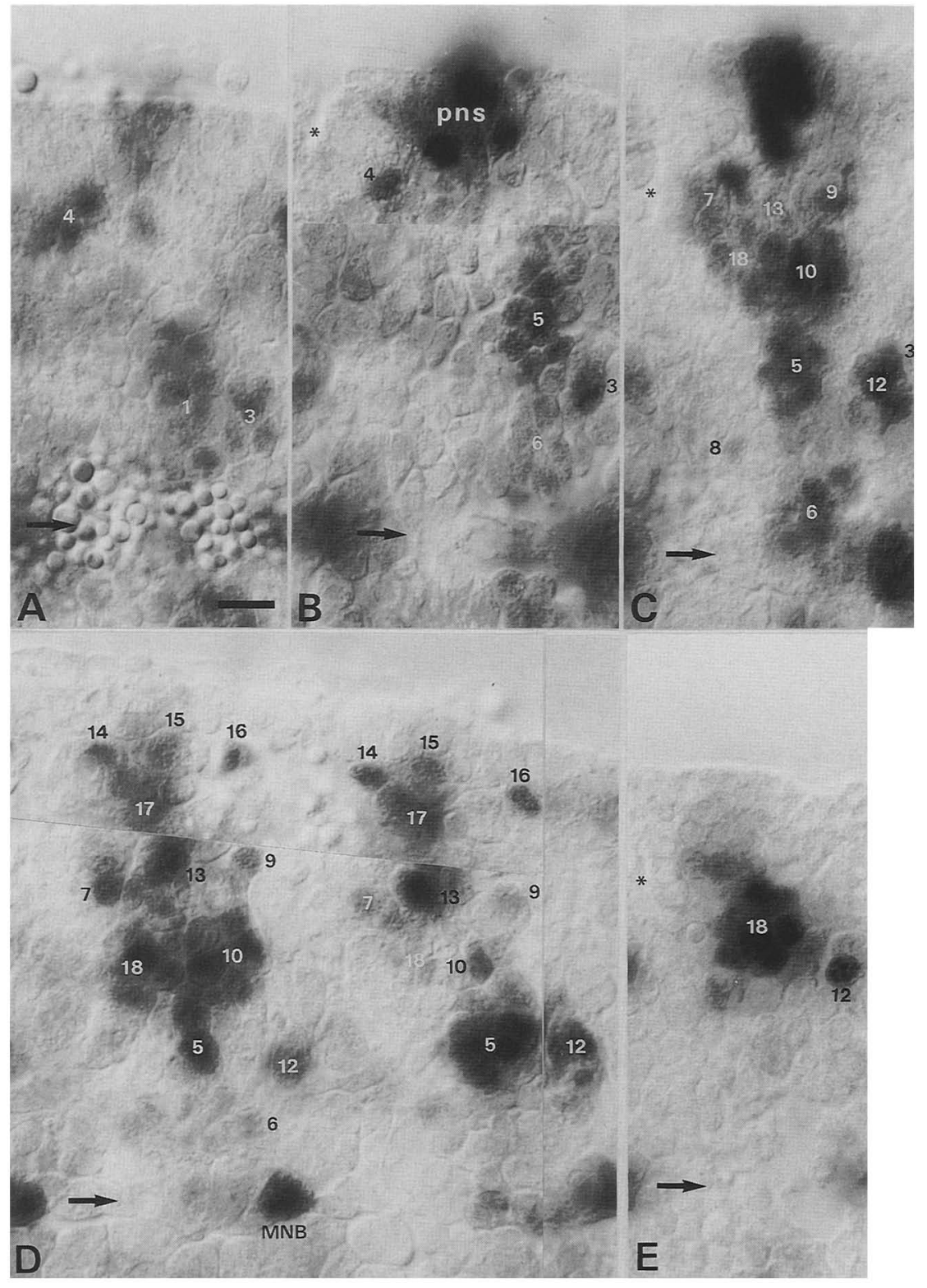

Figure 1. Successive stages in the mesodermal expression of l'sc. $(A, C, E)$ Single thoracic hemisegments; $(B)$ single abdominal hemisegment; $(D)$ third thoracic and first abdominal hemisegments. Earliest expression pattern shown is at late stage 10 in $A$. $(B-D)$ Progressively older embryos at stage 11. Numbers in white indicate the different clusters of l'sc expression, which at these stages still consist of several cells each (not all of which are in focus). Numbers in black indicate groups where expression is already restricted to a single cell or where a cell has clearly accumulated higher amounts of protein than the rest. Anterior is to the left; dorsal is up. Horizontal arrows indicate the ventral midline. (pns) A proneural cluster of the peripheral nervous system; (MNB) median neuroblast of the central nervous system. Asterisks (*) indicate the positions of tracheal pits. Bar, $10 \mu \mathrm{m}$. bryos with antibodies against L'sc and Twist. twist (twi) is initially expressed throughout the invaginated mesoderm (for review, see Bate 1993). After germ-band extension, twi is progressively lost from much of the mesoderm, persisting for a longer period in the somatic mesoderm, that is in cells that will give rise to the somatic muscles. This more restricted expression gives a segmental appearance to twi expression (Fig. 3A). In doublestained embryos we observe a close correlation between the mesodermal patterns of 1 'sc and twi expression (Fig. 3B). Furthermore, a detailed analysis at different stages indicates that all l'sc-expressing cells in the mesoderm express initially $t w i$, and that the progressive loss of $t w i$ in those cells precedes that of 1 'sc (not shown). Thus, we conclude that the clusters of l'sc-expressing cells are confined to the somatic muscle-forming mesoderm.
Single mesodermal cells expressing l'sc are muscle progenitor cells

To characterize further the evolving pattern of l'sc expression and in particular to attempt to identify those cells that are singled out within each cluster, we used the product of another gene, S59, which is expressed in three subsets (I-III) of muscle-forming cells, identified as muscle founder cells (Dohrmann et al. 1990). We chose S59 as a marker, because it is expressed sufficiently early in the embryonic mesoderm as to overlap with l'sc expression and to allow cells to be tested for coexpression of the two genes. S59 is coexpressed with l'sc in cells derived from three of the identified clusters of mesodermal l'sc expression (clusters 3, 10, and 13). In the case of groupings I'sc3/S59I and I'sc13/S59II, the cell that has 


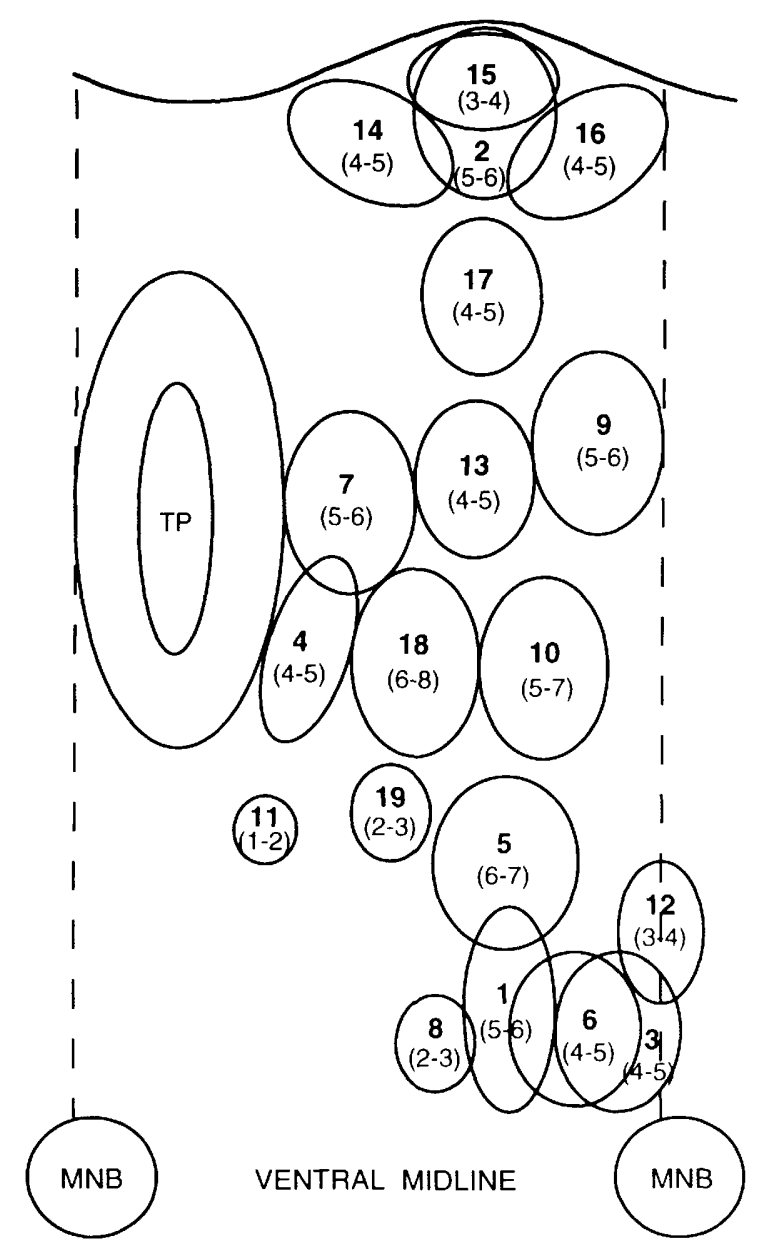

Figure 2. Diagram illustrating the relative positions of l'scexpressing cell clusters in the mesoderm. Clusters are numbered according to their approximate order of appearance. Number of cells per cluster is indicated in parentheses. In the case of clusters 1 and 2, l'sc expression begins in a dorsoventral stripe of $\sim 12$ cells, but it is then lost except from the ventral- or dorsalmost cells of each cluster, respectively. (TP) tracheal pit; (MNB) median neuroblast. Anterior is to the left; dorsal is up. Each cluster is shown at the position in the segment at which it arises. Because clusters arise in sequence as the segment develops, there are necessarily some distortions in the relationships shown between clusters in this diagrammatic representation.

accumulated higher amounts of L'sc and migrates to contact the ectoderm begins to coexpress $S 59$ (Fig. 3C,E). This cell rounds up and divides, at the same time losing l'sc expression, to give rise to two cells that are the founders of two distinct muscles (see below). In the case of grouping l'sc10/S59II the evolution of the pattern of coexpression is more complex. In this case, although l'sc also precedes $S 59$ expression, $S 59$ is initially expressed in all cells of cluster 10 (Fig. 3D). Thereafter, one cell is singled out, that is, it accumulates high levels of L'sc and S59 and comes to lie more superficially (Fig. 3D, inset a). As in the two previous cases, this cell divides, loses l'sc expression and produces two S59-expressing cells (Fig.
3D, inset b). Meanwhile, l'sc but not $S 59$ begins to disappear from most of the original cells in cluster 10. Later, a second cell, the last one of the cluster to retain l'sc expression, rounds up (Fig. 3D, inset b) and divides. 559 expression is now lost from all of the original cells in the cluster, persisting only in the four cells produced by the two divisions (not shown, see below).

We can summarize our observations as follows: Cells identified previously as prospective muscle founders by $S 59$ expression are derived from a distinct subset of l'scexpressing clusters. In each case, one or two cells of the cluster, which we call muscle progenitors, are singled out and coexpress I'sc and S59. The progenitors divide and each produces two cells that lose l'sc expression but maintain $S 59$ expression.

\section{The progeny of the 1'sc/S59-expressing cells}

We have looked in detail at the fates of the putative founder cells that are produced by the muscle progenitors that coexpress l'sc and S59. In the case of l'sc3/S59I, we confirm the description given by Dohrmann et al. (1990), who showed, for the two progeny of cell S59I (Fig. $4 \mathrm{~A})$, that cells Ia and Ib contribute to muscles 5 and 25, respectively (Fig. 4F). As Ia and Ib fuse with neighboring myoblasts to form the syncytial precursors of these muscles, these additional cells are recruited to 559 expression. However, $S 59$ expression continues at a high level only in muscle 25 and disappears from muscle 5 . In the case of l'sc10/S59II, our description differs significantly from that originally given by Dohrmann et al. (1990). In particular, we find that the four S59-expressing cells, produced by the division of the two progenitor cells from cluster 10, do not contribute solely to muscle 27 , as these authors suggest. Instead, these four cells contribute to four different muscle precursors (Fig. 4E). These are 26, 27, 29, and the ventral adult muscle precursor (Bate et al. 1991). We can identify these precursors with confidence on the basis of their position and their subsequent development. Only one of the cells maintains S59 expression at a high level as neighboring cells are recruited by fusion, this being the precursor of muscle 27 (Fig. 4F). There is transient expression at a lower level in the precursors of muscles 26 and 29 and, occasionally, we see expression at later stages in muscle 26. Expression is maintained at a low level in the adult muscle precursor, as it migrates to its final position between the segment borders. Our description of 1'sc13/S59III also differs from that given by Dohrmann et al. (1990), in that they describe $S 59$ expression as beginning in two cells, both of which contribute to muscle 18 . We find that $S 59$ expression begins in a single cell, a muscle progenitor (see above), and that this cell divides to give two S59expressing progeny (Fig. 4C,D). One of these cells is certainly the founder of muscle 18 (Fig, 4E,F). The second cell may be the founder of the adjacent muscle 11 , because it appears to remain distinct from the muscle 18 precursor and, in some instances, at later stages, we find S59 expression at low levels in muscle 11.

Thus we find that each cell produced by division of the 

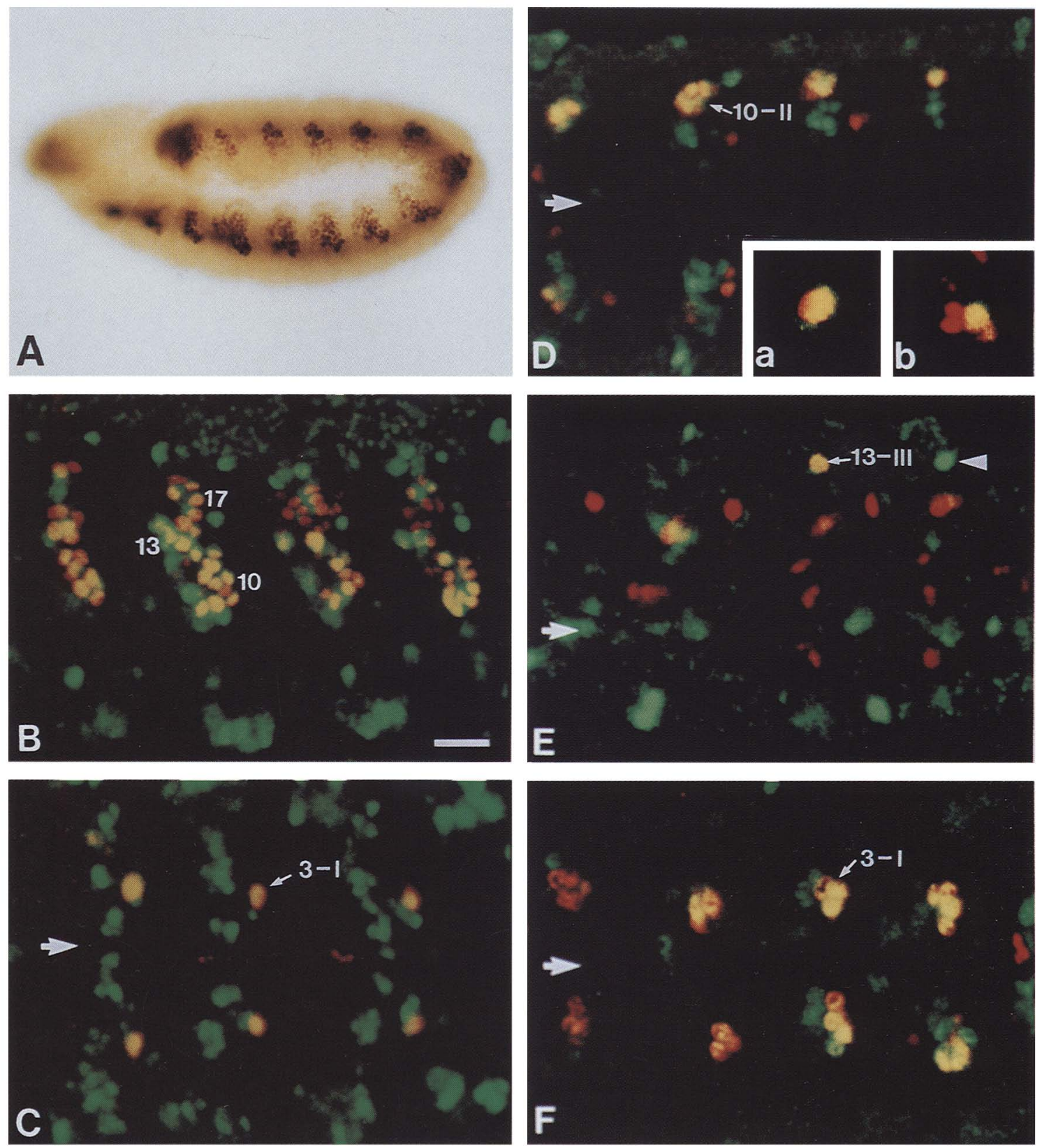

Figure 3. Coexpression of $l$ 'sc with $t w i$ and $S 59$ in the developing mesoderm. (A) Lateral view of a stage 11 embryo stained with anti-Twi antibody to show the characteristic distribution of $t w i$ expression in the mesoderm at this stage. $(B)$ Confocal micrograph showing a lateral view of an embryo at stage 11 double stained with anti-Twi (red) and anti-L'sc (green) antibodies. Cells that express the products of both genes are yellow. Numbers refer to the corresponding clusters of l'sc expression. Other cells that express only l'sc, for example, those located at the dorsal-most edge of the somatic mesoderm, had expressed $t$ wi at earlier stages. Cells in green at the bottom of the picture are located in the neuroectoderm. Small green dots at the top of the picture are background staining of the yolk. Bar, $20 \mu \mathrm{m}$. $(C-F)$ Ventral views of three to four segments of embryos at stage 11/12, double stained with antibodies against L'sc (green) and S59 (red). The arrow indicates the ventral midline. (C) Coexpression in the l'sc3/S59I progenitor. When l'sc expression becomes restricted to the cell that had accumulated higher levels of protein in cluster l'sc3, $S 59$ begins to be expressed in that cell. $|D|$ Coexpression in the l'sc10/S59II cluster prior to segregation of progenitors. (Inset a) Coexpression in the first progenitor of this group to segregate, lying more superficially than the rest of the cluster, which is found at a deeper plane of focus; (inset $b$ ) coexpression in the second progenitor, and the two progeny of the first progenitor expressing only S59. (E) Coexpression in the l'sc13/S59II progenitor. The cell (indicated by an arrowhead in one hemisegment) that has accumulated higher amounts of L'sc protein in cluster l'sc13 moves to a position more superficial than the rest of the cluster that still expresses l'sc (not shown). That cell soon begins to coexpress $S 59$, as already seen in another hemisegment (arrow). Cells closer to the midline, expressing $\$ 59$ alone, are derived from groups I and II or (more medial) are cells of CNS. (F) Coexpression in the l'sc3/S59I cluster in a DI mutant embryo. Several cells now express both genes, as opposed to only one in $C$. 
Figure 4. The development of the $S 59$ expression pattern in the somatic mesoderm of wild-type embryos. $(A-D)$ Ventral views of one to three segments of increasingly older embryos at stage 11 /early 12 . Roman numerals indicate the corresponding groups of 559 -expressing cells. Small arrows indicate the progenitors, which are larger than the rest. Large horizontal arrows point to the midline. Stained cells closer to the midline are S59-expressing cells in the CNS. $(A)$ Four S59I and one S59II groups are indicated. For S59I, in one case, the progenitor has not yet divided and, in the other three cases, the two progeny cells Ia and Ib are already present. For S59II, the group is at the initial cluster stage before the singling out of the first progenitor. $\{B \mid$ Four S59II groups at the same stage. The first progenitor is surrounded by smaller cells of the initial cluster. (C) In the lower S59II group the second progenitor is already present, adjacent to the two progeny of the first progenitor. The progenitor of one S59III group is also shown. $(D)$ The $S 59$ III progenitor has divided in two of the three segments shown; group II is represented either by clusters of four founders or two founders and a progenitor. The two founders derived from the group I progenitor are distinct at this stage. The muscle 5 founder is larger and more intensely stained than its sibling, the founder of muscle 25. $(E)$ Lateral view of a late stage 12 embryo showing $S 59$ expression in the precursors of the following muscles: 5 and 25 (the latter out of focus) derived from group S59I; 26, 27, 29, and in the adult ventral muscle precursor (A) from group

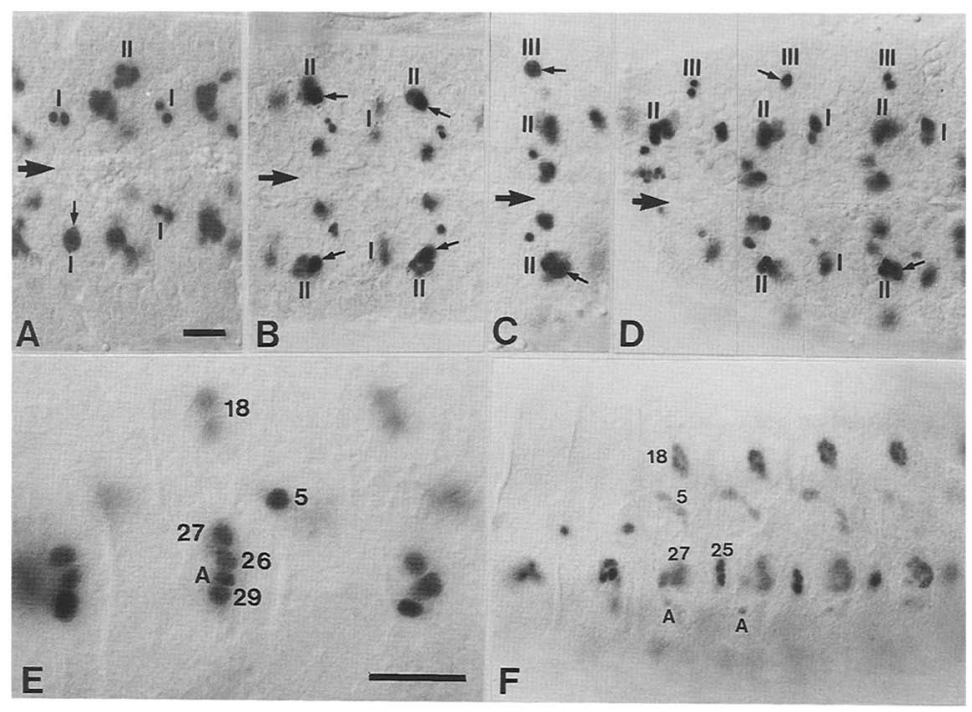
S59II; and 18 from group S59III. $(F)$ Lateral view of a stage 13 embryo. Muscle precursors that still express S59 are indicated. Bars, 20 $\mu \mathrm{m}$. (G) Diagram illustrating the progressive segregation of progenitors, founders and muscle precursors in the embryonic mesoderm. Progenitors segregate from clusters of $l$ 'sc-expressing cells and begin to express identity genes, i.e., $\$ 59$. Division of a progenitor gives rise to two founders which no longer express l'sc. Each founder recruits surrounding cells to form a syncytial muscle precursor.

S59-expressing muscle progenitors appears to be the founder of a distinct muscle. A diagram that summarizes our conclusions is shown in Figure 4G.

\section{1'sc and S59 expression in the mesoderm of neurogenic mutant embryos}

In neurogenic mutant embryos, the domains of mesodermal $S 59$ expression are expanded, suggesting that the small numbers of 559 -expressing cells in wild-type mesoderm are singled out from larger domains of potential S59-expressing cells (Bate et al. 1993). With this in mind, we looked at the expression of l'sc in the mesoderm of neurogenic mutant embryos to see whether the expansion of $S 59$ expression in such mutants coincides with a loss of the restriction of l'sc expression, which normally precedes $S 59$ expression in the wild type. In general, the process of singling out individual l'sc-expressing cells fails to occur in Delta $(D I)$ and Notch $(N)$ mutant embryos. Taking l'sc3/S59I as an example, we find that the normal restriction of 1 'sc expression does not take place and the entire cluster coexpresses l'sc and S59 (Fig. 3F). Thus we conclude that clusters of l'sc expression in the mesoderm of normal embryos represent domains of cells with the potential to express genes such as 559 . In the absence of neurogenic gene functions, singling out fails: l'sc expression remains throughout the cluster and each cell goes on to express $S 59$.

\section{The mesodermal phenotype of a deficiency for l'sc}

For the analysis of mutant phenotypes, we have focused on the 559 -expressing muscles and their precursors in $D f(1) s c^{19}$, a deficiency that removes l'sc. Muscles 27 and 18 are only rarely affected in the mutant embryos. In $26 \%\langle n=115\}$ of the mutant hemisegments, however, muscle 5 is abnormally formed, or it is not found in its normal position and an extra muscle with the morphology of muscle 8 appears instead (not shown). Furthermore, we also see some abnormalities in muscle 25 and, in many cases, it is missing altogether (Table 1 and Fig. $5 B \mid$. Because the founder cells of muscles 5 and 25 are the progeny of cell S59I, we looked at S59 expression in stage $11 D f(1) s c^{19}$ embryos. In $12 \%(n=164)$ of the cases no S59 expression is seen in cell I or its progeny (Fig. 5A), although we cannot say at present whether the cells fail to form or form but fail to express $S 59$. In another $20 \%$ of the cases the accumulation of $S 59$ is much lower than normal (data not shown). Thus, in at least $32 \%$ of the cases, the implementation of the myogenic pathway seems to be impaired at early stages, judging solely by the altered expression of S59. 
Table 1. Phenotype of muscle 25 in Df(1) $1 \mathrm{sc}^{19}$ embryos and its rescue by mesoderm-specific expression of l'sc

\begin{tabular}{|c|c|c|c|c|}
\hline \multirow[b]{2}{*}{ Genotype } & \multirow[b]{2}{*}{ Hemisegments $^{c}$} & \multicolumn{3}{|c|}{ Muscle 25} \\
\hline & & absent & abnormal $^{\mathrm{d}}$ & normal \\
\hline$D f(1 s c)^{19} / Y$ & $246(41)$ & $102(41.5 \%)$ & $18(7.3 \%)$ & $126(51.2 \%)$ \\
\hline Wild type & $222|37\rangle$ & $1(0.5 \%)$ & $6(2.7 \%)$ & $215(96.8 \%)$ \\
\hline$D f(1) s c^{19} / Y ;$ W381/UAS.l'sc ${ }^{b}$ & $264(44)$ & $32(12.1 \%)$ & $15(5.7 \%)$ & $217(82.2 \%)$ \\
\hline
\end{tabular}

a 'sc ${ }^{+}$embryos from the stock $D f(1) s c^{19} / F M 6$.

'W381 is a third chromosome insertion that expresses GAL4 uniformly in the mesoderm starting at stage 8. UAS.l'sc line 6 was used. 'Number of hemisegments and, in parentheses, of embryos examined. Abdominal hemisegments A2-A7 were scored in one-half of each embryo.

${ }^{\mathrm{d}}$ These muscles either have an abnormal morphology, do not express $S 59$, or show both defects.

We have also analyzed embryos of the $D f(1) s c^{4 L} s c^{9 R}$, another l'sc deletion. Such mutant embryos show a slightly stronger phenotype for muscles 5 and 25 (data not shown) than $D f(1) s c^{19}$ embryos. Because it is likely that there are deleterious factors mapping outside the deficiency which contribute to the enhanced phenotype of $D f(1) s c^{4 L} s c^{9 R}$ embryos (see also Martín-Bermudo et al. 1993 ), we only used $D f(1) s c^{19}$ for further experiments.

\section{Mesodermal expression of l'sc rescues mutant phenotypes in muscles}

Because l'sc is expressed in the neuroectoderm, neuroblasts, and the mesoderm, and effects in the mesoderm of mutations in l'sc might be secondary consequences of a derangement in the ectoderm or in the central nervous system (CNS), we used the GAL4 targeted expression system (Brand and Perrimon 1993) to promote l'sc expression in the mesoderm of $D f(1) s c^{19}$ embryos. We used the G4.W381 line, which promotes homogeneous GAL4 expression exclusively in the mesoderm from stage 8 onward, to drive UAS.l'sc. The level of l'sc expression under these conditions aproximates the level of endogenous l'sc expression in the wild type. We scored for phenotypes in $S 59$ group I at early stages and in muscle 25 at late stages, these being the most strongly affected by loss of l'sc. In the case of cell S59I and its progeny, rescue of the l'sc phenotype is virtually complete; we find defec-

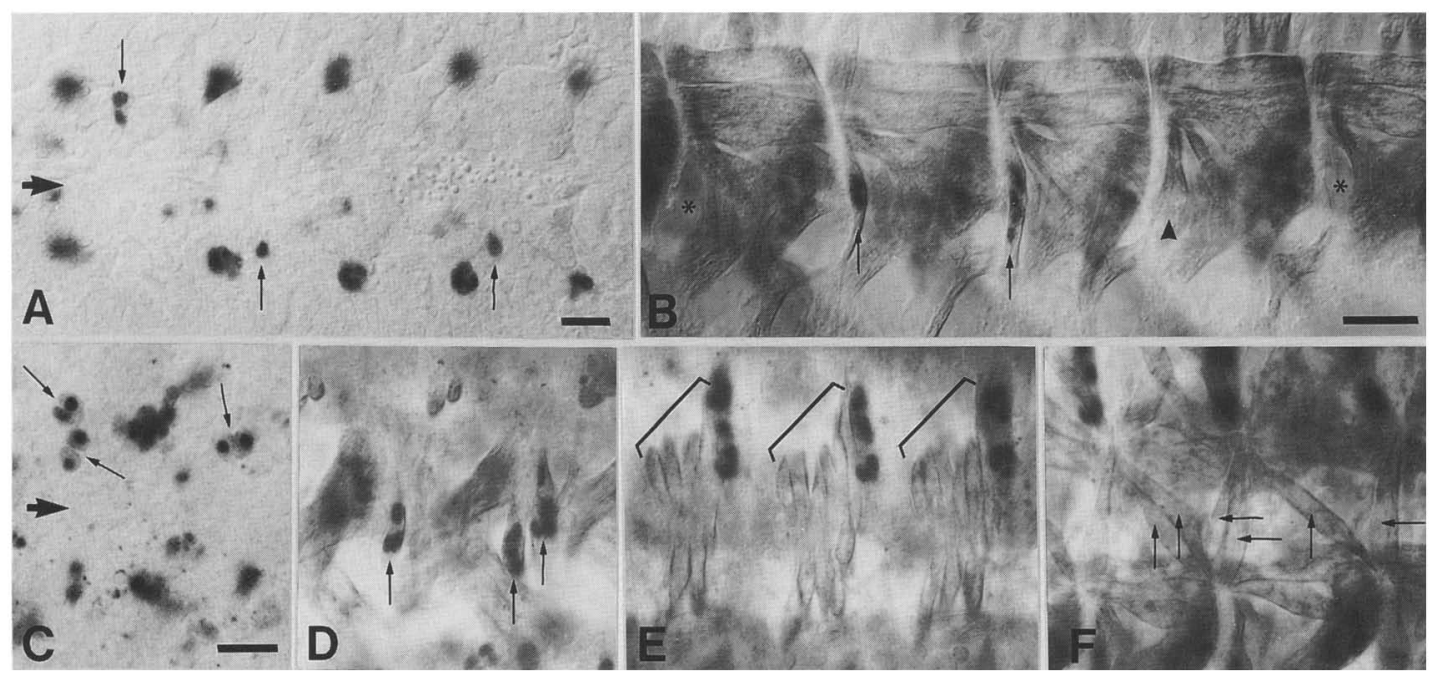

Figure 5. Mesodermal phenotypes produced by the absence and by the excess of l'sc function. $(A, B)$ Phenotypes of $D f(1) s c^{19}$ embryos. $(C-E)$ Phenotypes of normal embryos which, in addition to the endogenous l'sc expression, also express the gene throughout the mesoderm under the control of GAL4. Anterior is to the left. $(A, C)$ Ventral views of stage 11 embryos stained with anti-S59 (horizontal arrows indicate ventral midline). $(B, D-F)$ Lateral views of stage 16 embryos double stained with anti-S59 and the muscle specific antibody 3E2. (A) Vertical arrows point to those S59I founder cells which are present in a $D f(1) s c^{19}$ embryo: One hemisegment has both, two have only one and both cells are missing, or not expressing $S 59$, in the rest. $(B)$ Arrows point to normal examples of muscle 25 in a $D f(1) s c^{19}$ embryo; the arrowhead indicates a muscle 25 that fails to express $S 59$; asterisks ( $\left.{ }^{*}\right)$ indicate the expected locations of muscle 25 where it is missing. $(C)$ Duplication of the two S59I founders cells (arrows) in one hemisegment. (D) Duplication of muscle 25 (arrows) in one hemisegment. (E) Duplications in the cluster of muscles 21-24 (brackets): The left-most hemisegment has the normal complement of four muscles; the other two hemisegments have six muscles in the cluster. $|F|$ Duplications of muscle 5 (vertical arrows) and muscle 8 (horizontal arrows) in one hemisegment. 
tive 559 expression in only $1.5 \%(n=136)$ of the mutant hemisegments. This is accompanied by a substantial rescue of the muscle 25 phenotype (Table 1). There are a number of reasons why the rescue that we observe for the muscle may be incomplete, including the fact that CNS defects, that are not rescued in these embryos, may interfere non-specifically with the normal development of muscle 25. Nonetheless, the fact that mesodermal expression of 1 'sc can substantially rescue both $S 59$ expression and the muscle 25 phenotype strongly supports the notion that there is a direct requirement for l'sc expression in the mesoderm and that this function of l'sc may be involved in the proper partitioning of the mesoderm into muscle-forming groups. This view is reinforced by the observation that in sibling embryos (i.e., embryos carrying an endogenous l'sc gene, in addition to the l'sc expression driven by GAL4) there is a variable phenotype in which individual muscles are duplicated (Fig. 5D-F) and occasional duplications of S59-expressing progenitors or founder cells can be observed (Fig. 5C). To improve the yield of muscle duplications, we crossed G4.SG30 females that carry two GAL4 insertions in homozygous condition to males homozygous for a second chromosome carrying two UAS.l'sc insertions (line 4 plus line 10) (see Materials and methods), and examined abdominal hemisegments A1-A6 in 10 embryos. Under those conditions, most hemisegments analyzed (53/60) show between 1 and 6 extra muscles, resulting, on average, in $2(123 / 60)$ duplicated muscles per hemisegment.

\section{Discussion}

\section{Muscle progenitors and muscle founders}

Our observations suggest that muscle founder cells are derived by division from cells that we designate as muscle progenitors. Progenitors, in the cases we have described, are distinctive cells that segregate from clusters of l'sc-expressing cells.

The mesodermal phenotype of mutations in the neurogenic genes (Corbin et al. 1991; Bate et al. 1993) suggests that the definition of muscle progenitor cells in the mesoderm requires a prior demarcation of small clusters of cells from which progenitors are singled out by a competitive interaction with their neighbors. In the nervous system, expression of proneural genes defines the cell groups from which neural progenitors arise, and it is obviously of interest to discover how such groups are defined in the somatic mesoderm. Like the proneural AS-C genes in neurogenesis, the localized expression of the "promuscle" genes would impose a spatial organization on the process of myogenesis to initiate the formation of muscle progenitors. One possibility of course is that the proneural genes, or a subset of them, function in the mesoderm as in the ectoderm to define cell clusters from which muscle progenitors will be singled out.

\section{l'sc and the segregation of muscle progenitors}

This is the context in which we have looked at the mesodermal expression of the proneural genes of the AS-C.
We find that l'sc is expressed in the mesoderm. Furthermore, it is expressed in clusters of cells in the somatic, muscle-forming mesoderm. These clusters appear in sequence, prior to the segregation of muscle founders. The pattern of l'sc expression evolves, such that a single cell accumulates higher levels of protein. With $S 59$ as a marker for some muscle-forming cells, we find that the cell singled out from the l'sc cluster by higher levels of expression is also the cell that becomes the muscle progenitor.

The idea that the clusters of l'sc-expressing cells represent the groups of equivalent cells from that individual muscle progenitor cells segregate is reinforced by the patterns of expression which we observe in $D l$ and $N$ mutant embryos. In the cluster that we have studied in detail, the restriction of l'sc expression to a single cell fails to occur in such embryos. Instead, all cells in the cluster retain l'sc expression and each of these cells expresses S59. Thus the limits to the group of equivalent cells with the potential to express $S 59$ coincide with the boundaries of cell clusters defined by l'sc expression. Therefore, l'sc seems to demarcate the group of equivalent cells from which a muscle progenitor cell will be singled out.

If we combine our observations with previous analyses of myogenesis in Drosophila, we can synthesize the early phases of the muscle patterning process as follows. l'sc is activated in clusters of cells, conferring on these cells the potential to form muscle progenitors. This potential is then localized to a single cell, sometimes two, within the cluster by a process of competitive interaction that is mediated by the products of the neurogenic genes. This cell moves into close contact with the ectoderm and divides to give rise to two cells, both of which have the characteristics of muscle founder cells.

We note that the ventral adult muscle precursor (Bate et al. 1991) is generated at the same time and in exactly the same way as its siblings, the founders of larval muscles. This is a new observation reinforcing the idea that the specification of many adult muscles occurs in the embryo as part of a single patterning process that organizes the larval and adult musculature (Bate 1993).

The process that we outline leads to the acquisition of the general characteristics common to all muscle progenitor cells and their progeny, the muscle founder cells. Additional information must be required to endow each of these cells with the special characteristics that lead to the formation of distinct, different muscles. In the cases that we have studied, we have used $S 59$ as a marker, and it has been proposed that $S 59$ is a gene of this second kind (Dohrmann et al. 1990). We can draw an analogy between genes of this class and neuroblast identity genes, which are involved in the specification of individual neural progenitors (for review, see Goodman and Doe 1993). In both cases, the expression of genes of this identity class is usually preceded by the clustered expression of genes of the proneural type, and in both cases identity genes are expressed either in a single progenitor cell, or in all cells of the cluster from which this progenitor will arise. l'sc cluster 10 is perhaps unusual in that it gives 
rise to two muscle progenitor cells. We note, however, that proneural clusters in the ectoderm of the imaginal discs may give rise to more than one progenitor (Cubas et al. 1991).

\section{l'sc and muscle patterning}

The fact that l'sc is expressed in the cell clusters from which muscle progenitors arise suggests, but does not prove, that $l$ 'sc has a function in the early patterning of the somatic mesoderm. Nonetheless, the observation that the lack of $l$ 'sc deranges the development of one of the S59-expressing progenitors and the muscles that are derived from it, points strongly to a requirement for l'sc in this early patterning process. Because overexpression of l'sc leads to a duplication of progenitors and muscles, we argue that it functions in the generation of the muscle progenitors themselves. Furthermore, the fact that mesodermal-restricted expression of l'sc rescues the 559 phenotype and largely rescues the muscle phenotype of a l'sc deficiency confirms that the requirement for l'sc in the mesoderm is direct and not an indirect consequence of some failure in ectodermal patterning or in early neurogenesis. The fact that duplication phenotypes are limited in number may have a plausible explanation in the lateral inhibition exerted by the normally arising muscle progenitors, which are present at a relatively high density. In agreement with this hypothesis, GAL4-directed l'sc overexpression in the embryonic neuroectoderm is not apparently able to induce the formation of extra neuroblasts, given the high density of proneural clusters in the neuroectoderm, and consequently of neuroblasts developing from those clusters (B. Giebel, I. Stuettem, U. Hinz, and J.A. Campos-Ortega, pers. comm.), although it promotes the formation of a substantial number of extra sensory organ mother cells in imaginal discs, where the density of proneural clusters is much lower (Hinz et al. 1994).

Clearly the phenotype we observe for loss of l'sc function is far weaker than we might have predicted on the basis of its expression pattern alone. This lack of correlation between phenotype and expression, however, is also characteristic of l'sc in its well-documented role as a proneural gene in the developing CNS. In the neuroectoderm, l'sc is expressed in the proneural clusters that give rise to many neuroblasts (Martín-Bermudo et al. 1991). Nevertheless, only a small fraction of these neuroblasts are affected by deficiencies that remove l'sc, and this has led to the suggestion that other genes, with similar functions, can partially substitute for 1'sc /Cabrera et al. 1987; Jiménez and Campos-Ortega 1990; Martín-Bermudo et al. 1991). The existence of maternal contribution of l'sc that might mask mutant phenotypes has been excluded previously (Jiménez and Campos-Ortega 1982). Therefore, we assume that, as in the CNS, there is some degree of functional redundancy in the process that leads to the formation of muscle progenitors. Genes that could cooperate with l'sc in this function may not necessarily encode bHLH proteins nor have a broad pattern of expression like l'sc, but they are expected to produce sim- ilar mutant phenotypes. One such gene could be apterous $(a p)$, a putative identity gene expressed in a subset of muscles (Bourgouin et al. 1992). ap lack-of-function and overexpression phenotypes are similar in nature and expressivity to those reported here for l'sc (Bourgouin et al. 1992). Another candidate to cooperate with l'sc in the generation of muscle progenitors could be daughterless (da), which encodes a bHLH protein known to interact with the L'sc protein (Murre et al. 1989; Cabrera and Alonso 1991). In addition, da mutants display a muscle phenotype (Caudy et al. 1988). Our preliminary analysis of $d a$ mutants indicates that the segregation of muscle progenitors, as defined by accumulation of higher levels of L'sc and movement towards the ectoderm, seems to be normal; however, 559 expression is altered. This is analogous to the situation in the nervous system, where $d a$ is essential for neural precursor differentiation but not for the initiation of neural precursor formation (Jiménez and Campos-Ortega 1990; Vaessin et al. 1994). In vertebrate myogenesis, there are similar indications of functional redundancy. Thus, for example, loss of function in myf5 or myo $D$ has remarkably mild phenotypic consequences in the muscles, whereas the double knockout of both genes produces mice in which myoblasts and differentiated muscles fail to form (Braun et al. 1992; Rudnicki et al. 1992, 1993).

It seems likely that the clusters of l'sc expression that we have described are sufficient to account for the cell groupings from which the founders of each of the 30 different muscles in the hemisegment will segregate. Thus the expression of the L'sc protein may be common to the progenitors of all the embryonic muscles and represent an early stage in the pathway leading to terminal muscle differentiation. By analogy with the CNS (Martín-Bermudo et al. 1993), the pattern of l'sc expression in the somatic mesoderm is likely to depend on multiple cis-acting regulatory elements, and it is this positionally regulated expression which, together with the expression of other, so-far-unidentified genes, imposes a spatial organization on the initial phases of muscle formation.

\section{An analogy between myogenesis and neurogenesis}

The apparent role of the proneural gene l'sc in mesodermal development suggests that, despite obvious differences, there might be an underlying similarity in the generation of muscles and of the neurons that will innervate them. Indeed, the events leading to the formation of muscle founder cells are analogous to the development of midline precursor (MP) neurons in the CNS (Bate and Grunewald 1981; Thomas et al. 1984). The sequence by which MPs are generated from a progenitor, which is singled out from a cluster and divides to give rise to two neural cells, may be the basic scheme of proliferation that produces neurons in the CNS. The introduction in most lineages of a repetitively dividing blast cell, the neuroblast, produces a sequence of neural progenitors (ganglion mother cells) from one original proneural cluster, thus introducing additional complexity into the network, which ultimately regulates the opera- 
tion of the muscles. In both tissues, however, it is the segregation of a progenitor, or sequence of progenitors, which then divide to give rise to sibling cells with different fates, which generates the elements of the neural and muscle patterns.

Like the neurons that innervate them, however, the Drosophila muscles are a highly differentiated set, and this is reflected in the subsequent expression of different transcription factors in the muscle progenitors and founder cells. The challenge is now to identify the additional elements of the myogenic pathway, which, together with l'sc, promote the development of mesodermal cell clusters from which muscle progenitors and the founders of individual muscles will be formed.

\section{Materials and methods}

Fly strains and crosses

Flies were raised at $25^{\circ} \mathrm{C}$ on standard medium. Strains used were as follows: Oregon-R; $D f(1) s c^{19}$, a partial deletion of the AS-C that removes the $a c, s c$ and l'sc genes; $D f(1) s c^{4 L} s c^{9 R}$, a synthetic deficiency obtained by recombination of two inversions, which only removes the l'sc transcription unit; $N^{55 e 11}$ and $D I^{F X 3}$ (Lindsley and Zimm 1992). G4.W381 (from J. Urban, University of Mainz, Germany) is a homozygous viable third chromosome insertion which expresses the GAL4 gene in the mesoderm at germ-band extension. G4.SG30 (from A. Michelson, Howard Hughes Medical Institute, Boston, MA) is a strain that contains the homozygous viable, third chromosome G4.24B insertion (Brand and Perrimon 1993; Michelson 1994) plus an X chromosome insertion of GAL4 under the control of the twi promoter (from S. Greig and M. Akam, Wellcome/CRC Institute, Cambridge, UK). The UAS.l'sc strains were constructed as follows: a Dra-Dra genomic fragment containing the coding sequence of l'sc (Martín-Bermudo et al. 1993) was cloned into the pUAST vector (Brand and Perrimon 1993) and used to transform white flies, following standard protocols. Several stable transgenic lines were established, and three of them, lines 6 (a third chromosome insertion), 4, and 10 (two independent second chromosome insertions recombined in the same chromosome), were used in the present study.

For the analysis of mutant phenotypes of $D f(1) s c^{19}$ embryos, we used the stock $D f(1) s c^{19} / F M 6$. Stage 11 mutant embryos were identified by lack of l'sc expression using a L'sc antibody. Stage 15-16 mutant embryos were recognized by their hypoplasic CNS phenotype. For the rescue experiment with the GAL4 system $D f(1) s c^{19} / F M 6$; UAS.l'sc line 6 females were mated to G4.W381 males; rescued $D f(1) s c^{19}$ embryos were recognized by the lack of endogenous l'sc expression in the neuroectoderm at stage 11 or by their mutant CNS at stages 15-16.

\section{Immunocytochemistry}

The following primary antibodies were used: rat anti-L'sc (Martín-Bermudo et al. 1991), anti-Twist (Roth et al. 1989), mAb 3E2, which stains all muscles (from A. Ferrús, Instituto Cajal, Madrid, Spain) and anti-S59. Anti-S59 was raised in rats and rabbits by immunization with a purified glutathione fusion protein containing a 924-bp BamHI 559 fragment (Dohrmann et al. 1990). This fragment does not encode the homeo domain, but the fusion protein is recognized by the preexisting polyclonal anti-S59 antibody provided by M. Frasch (Mount Sinai School of Medicine, NY). The resulting sera were tested for specificity on wild-type embryos and by comparing the pattern of staining with previously published data for the expression of the S59 protein (Dohrmann et al. 1990).

Embryos raised at $25^{\circ} \mathrm{C}$ were fixed and stained as described previously (Martín-Bermudo et al. 1991). Stages are according to Campos-Ortega and Hartenstein (1985). For single-labeling experiments we used a biotinylated secondary antibody in combination with a Vectastain Elite kit (Vectorlabs). For doublelabeling experiments, following simultaneous incubation with the primary antibodies, embryos were treated with the first species-specific biotinylated secondary antibody and the Elite kit. After staining for peroxidase (brown precipitate), the embryos were treated in the same way with the second species-specific biotinylated antibody and the Elite kit, and the staining reaction for peroxidase was carried out in the presence of $\mathrm{Ni}$ and $\mathrm{Co}$ ions (blue precipitate). Occasional stained embryos were dissected and embedded in Epon resin. For immunofluorescence a similar protocol was followed, in which a lissamine rhodamine (LRSC)labeled secondary antibody was used to detect binding of S59 or Twi primary antibodies. For the detection of L'sc antibody, we used a biotinylated secondary antibody and fluorescein (FITC)labeled streptavidin. Fluorescent images were recorded by use of a Zeiss LSM 310 confocal microscope.

\section{Acknowledgments}

We thank S. de la Escalera and S. Romani for their help in obtaining the UAS.l'sc strains; M. Baylies and E. Rushton for the S59-fusion protein; J. Urban and A.M. Michelson for flies; S. Roth and A. Ferrús for antibodies; and J.A. Campos-Ortega for communicating unpublished results. We also thank S. Campuzano, J. Modolell, M. Baylies, and M. Ruiz Gómez for their helpful comments on the manuscript. A.C. is a predoctoral fellow of Ministerio the Educación y Ciencia. This work was supported by a grant from DGICYT (to F.J.), an institutional grant from Fundación Ramón Areces, and a grant from the Wellcome Trust (to M.B.).

The publication costs of this article were defrayed in part by payment of page charges. This article must therefore be hereby marked "advertisement" in accordance with 18 USC section 1734 solely to indicate this fact.

\section{References}

Bate, M. 1990. The embryonic development of larval muscles in Drosophila. Development 110: 791-804.

- 1993. The mesoderm and its derivatives. In The development of Drosophila melanogaster. (ed. M. Bate and A. Martínez Arias|, pp. 1013-1090. Cold Spring Harbor Laboratory Press. Cold Spring Harbor, New York.

Bate, M. and E.B. Grunewald. 1981. Embryogenesis of an insect nervous system. II. A second class of precursor cells and the origin of the intersegmental connectives. I. Embryol. Exp. Morphol. 61: 317-330.

Bate, M., E. Rushton, and D.A. Currie. 1991. Cells with persistent $t$ wist expression are the embryonic precursors of adult muscles in Drosophila. Development 113: 79-89.

Bate, M., E. Rushton, and M. Frasch. 1993. A dual requirement for neurogenic genes in Drosophila myogenesis. (Suppl.) Development 149-161.

Bour, B.A., M.A. O'Brien, W.L. Lockwood, E.S. Goldstein, R. Bodmer, P.H. Taghert, S.M. Abmayr, and H.T. Nguyen. 1995. Drosophila MEF2, a transcription factor that is essential for myogenesis. Genes \& Dev. 9: 730-741.

Bourgouin, C., S.E. Lundgren, and J.B. Thomas. 1992. apterous is 
a Drosophila LIM domain gene required for the development of a subset of embryonic muscles. Neuron 9: 549-561.

Brand, A.H., and N. Perrimon. 1993. Targeted gene expression as a means of altering cell fates and generating dominant phenotypes. Development 118: 401-415.

Braun, T., M.A. Rudnicki, H.H. Arnold, and R. Jaenisch. 1992. Targeted inactivation of the muscle regulatory gene Myf5 results in abnormal rib development and perinatal death. Cell 71: 369-382.

Cabrera, C.V. and M.C. Alonso. 1991. Transcriptional activation by heterodimers of the achaete-scute and daughterless gene products of Drosophila. EMBO J. 10: 2965-2973.

Cabrera, C.V., A. Martínez Arias, and M. Bate. 1987. The expression of three members of the achaete-scute gene complex correlates with neuroblast segregation in Drosophila. Cell 50: 425-433.

Campos-Ortega, J.A. 1993. Early neurogenesis in Drosophila melanogaster. In The development of Drosophila melanogaster. (ed. M. Bate and A. Martínez Arias), pp. 1091-1129. Cold Spring Harbor Laboratory Press, Cold Spring Harbor, New York.

Campos-Ortega, J.A. and V. Hartenstein. 1985. The embryonic development of Drosophila melanogaster. Springer-Verlag, Berlin, Germany.

Caudy, M., E.H. Grell, C. Dambly-Chaudiére, A. Ghysen, L.Y. Jan, and Y.N. Jan. 1988. The maternal sex determination gene daughterless has zygotic activity necessary for the formation of peripheral neurons in Drosophila. Genes \& Dev. 2: $843-852$.

Corbin, V., A.M. Michelson, S.M. Abmayr, V. Neel, E. Alcamo, T. Maniatis, and M. Young. 1991. A role for the Drosophila neurogenic genes in mesoderm differentiation. Cell 67: $311-$ 323.

Cubas, P., J.-F. de Celis, S. Campuzano, and J. Modolell. 1991. Proneural clusters of achaete-scute expression and the generation of sensory organs in the Drosophila imaginal wing disc. Genes \& Dev. 5: 996-1008.

Davis, R., H. Weintraub, and A. Lassar. 1987. Expression of a single transfected cDNA converts fibroblasts to myoblasts. Cell 51: 987-1000.

Dohrmann, C., N. Azpiazu, and M. Frasch. 1990. A new Drosophila homeo box gene is expressed in mesodermal precursor cells of distinct muscles during embryogenesis. Genes \& Dev. 4: 2098-2111.

Goodman, C.S. and C.Q. Doe. 1993. Embryonic development of the Drosophila central nervous system. In The Development of Drosophila melanogaster. (ed. M. Bate and A. Martínez Arias|, pp. 1131-1206. Cold Spring Harbor Laboratory Press, Cold Spring Harbor, New York.

Hinz, U., B. Giebel, and J.A. Campos-Ortega. 1994. The basichelix-loop-helix of Drosophila lethal of scute protein is sufficient for proneural function and activates neurogenic genes. Cell 76: 77-87.

Jan, Y.N. and L.Y. Jan. 1993. HLH proteins, fly neurogenesis and vertebrate myogenesis. Cell 75: 827-830.

Jiménez, F. and J.A. Campos-Ortega. 1982. Maternal effects of zygotic mutants affecting early neurogenesis in Drosophila. Roux's Arch. Dev. Biol. 191: 191-201.

- 1990. Defective neuroblast commitment in mutants of the achaete-scute complex and adjacent genes of Drosophila melanogaster. Neuron 5: 81-89.

Lilly, B., B. Zhao, G. Ranganayakulu, B.M. Paterson, R.A. Schulz, and E.N. Olson. 1995. Requirement of MADS domain transcription factor D-MEF2 for muscle formation in Drosophila. Science 267: 688-693.

Lindsley, D. and G.G. Zimm. 1992. The genome of Drosophila melanogaster. Academic Press Inc., New York.

Martín-Bermudo, M.D., C. Martínez, A. Rodríguez, and F. Jiménez. 1991. Distribution and function of the lethal of scute gene product during early neurogenesis in Drosophila. Development 113: 445-454.

Martín-Bermudo, M.D., F. Gonzalez, M. Domínguez, I. Rodríguez, M. Ruiz-Gómez, S. Romani, J. Modolell, and F. Jiménez. 1993. Molecular characterization of the lethal of scute genetic function. Development 118: 1003-1012.

Michelson, A.M. 1994. Muscle pattern diversification in Drosophila is determined by the autonomous function of homeotic genes in the embryonic mesoderm. Development 120: $755-768$.

Michelson, A.M., S. Abmayr, M. Bate, A. Martinez Arias, and T. Maniatis. 1990. Expression of a MyoD family member prefigures muscle pattern in Drosophila embryos. Genes \& Dev. 4: 2086-2097.

Murre, C., P. Schonleber McCaw, H. Vaessin, M. Caudy, L.Y. Jan, Y.N. Jan, C.V. Cabrera, J.N. Buskin, S.D. Hauschka, A.B. Lassar, H. Weintraub, and D. Baltimore. 1989. Interactions between heterologous helix-loop-helix proteins generate complexes that bind specifically to a common DNA sequence. Cell 58: 537-544.

Paterson, B.M., U. Walldorf, J. Eldridge, A. Dubendorfer, M. Frasch, and W. Gehring. 1991. The Drosophila homologue of vertebrate myogenic determination genes encodes a transiently expressed nuclear protein marking primary myogenic cells. Proc. Natl. Acad. Sci. 88: 3782-3786.

Roth, S., D. Stein, and C. Nüsslein-Volhard. 1989. A gradient of nuclear localization of the dorsal protein determines dorsoventral pattern in the Drosophila embryo. Cell 59: 11891202.

Rudnicki, M.A., T. Braun, S. Hinuma, and R. Jaenisch. 1992. Inactivation of $M y O D$ in mice leads to up-regulation of the myogenic HLH gene Myf5 and results in apparently normal muscle development. Cell 71: 383-390.

Rudnicki, M.A., P.N.J. Schnegelsberg, R.H. Stead, T. Braun, H.H. Arnold, and R. Jaenisch. 1993. MyoD or Myf5 is required for the formation of skeletal muscle. Cell 75: 13511359.

Rushton, E., R. Drysdale, S.M. Abmayr, A.M. Michelson, and M. Bate. 1995. Mutations in a novel gene, myoblast city, provide evidence in support of the founder cell hypothesis for Drosophila muscle development. Development 121: 1979-1988.

Thomas, J.B., M.J. Bastiani, C.M. Bate, and C.S. Goodman. 1984. From grasshopper to Drosophila: A common plan for neuronal development. Nature 310: 203-207.

Vaessin, H., M. Brand, L.Y. Jan, and Y.N. Jan. 1994. daughterless is essential for neuronal precursor differentiation but not for initiation of neuronal precursor formation in Drosophila embryo. Development 120: 935-945.

Williams, J.A., J.B. Bell, and S.B. Carroll. 1991. Control of Drosophila wing and haltere development by the nuclear vestigial gene product. Genes \& Dev. 5: 2481-2495. 


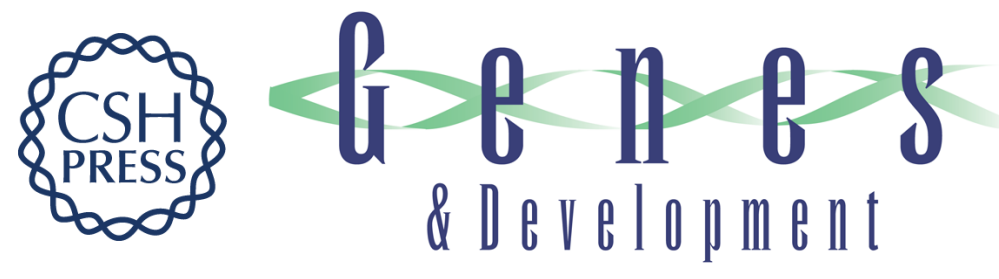

\section{Lethal of scute, a proneural gene, participates in the specification of muscle progenitors during Drosophila embryogenesis.}

A Carmena, M Bate and $F$ Jiménez

Genes Dev. 1995, 9:

Access the most recent version at doi:10.1101/gad.9.19.2373

References This article cites 32 articles, 16 of which can be accessed free at:

http://genesdev.cshlp.org/content/9/19/2373.full.html\#ref-list-1

License

Email Alerting

Service

Receive free email alerts when new articles cite this article - sign up in the box at the top right corner of the article or click here.

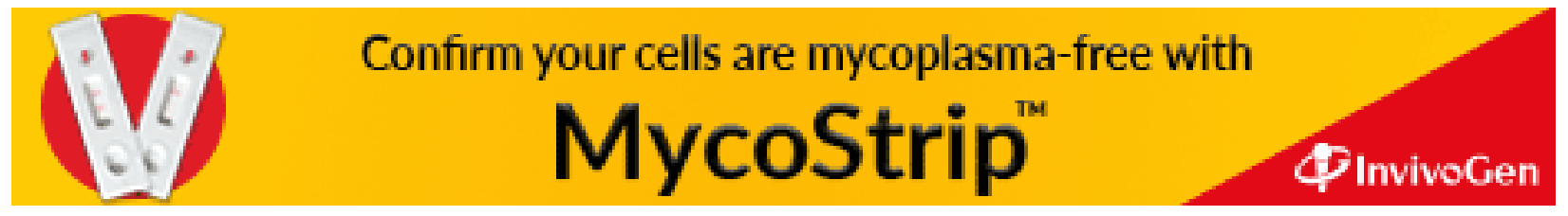

